\title{
Development of a clinical scale to assess retropulsion in neurological disorders
}

Neurol Rehabil 2019;25:S7-S17 (C) Hippocampus Verlag 2019

DOI: $10.14624 /$ NR1904002

\author{
J. Bergmann ${ }^{1,2}$, C. Krewer ${ }^{1,3}$, E. Koenig'2, F. Müller ${ }^{1,2}$, K. Jahn $^{1,2}$ \\ 1 German Center for Vertigo and Balance Disorders (DSGZ), Ludwig-Maximilians University of Munich, \\ Germany \\ 2 Schoen Clinik Bad Aibling, Bad Aibling, Germany \\ 3 Technical University Munich, Institute for Sport and Health Science, Human Movement Science \\ Department, Munich, Germany
}

\section{Introduction and background}

Retropulsion reflects a disturbed postural alignment in the sagittal plane, similar to lateropulsion in the frontal plane. It is characterized by a spontaneous posterior body tilt with the risk of backward falling, active backward pushing with the inability to shift the centre of mass forward, and resistance against passive correction. So far, research on this postural behavior is very limited, even though it seems quite frequent and relevant for neurorehabilitation [9, 14]. Unpublished data of a short survey among 22 therapists at our clinic showed that they are frequently confronted with retropulsion during their work and that the postural behavior hampers the therapy and negatively affects the rehabilitation process. One major problem in the investigation of retropulsion is that there was hitherto no established tool available to rate its severity. As far as we know, among existing scales for postural control, only the Backward Disequilibrium Scale qualitatively evaluates deficient postural control in the sagittal plane [10]. The Backward Disequilibrium Scale assesses the posterior position of the center of mass during five different tasks, but does not assess whether subjects actively shift their center of mass backward and whether they show resistance against passive correction. In addition, the clinimetric properties of the scale are insufficiently investigated and the scale did not make its way into clinical and research practice.

Consequently, an established clinical scale to evaluate retropulsion is urgently needed for clinical work, but also to study the epidemiology, the etiology, and the rehabilitation process of subjects with retropulsion. That's why the objective of this study was to develop a clinical scale to quantify retropulsion.

\section{Methods}

A preliminary version of the Scale for Retropulsion (Scale for Retropulsion-Vo) has been set up by an interdisciplinary team at the Schoen Clinic Bad Aibling. This scale was further developed in a Delphi study.
Delphi study

The Delphi method was employed to gather opinions from experts and build consensus on the validity of the content of the Scale for Retropulsion [6]. Three rounds were intended for the Delphi study. During each round, the experts were invited to respond to specific questions in an online survey. The online tool LimeSurvey (LimeSurvey $\mathrm{GmbH}$, Hamburg, Germany) was used to design and answer the surveys. The surveys included statements with instructions to indicate the level of agreement or disagreement by using a nine-point Likert scale ranging from strongly disagree (1 point) to strongly agree (9point), and free space for the experts to explain their rating on the Likert scale and to provide comments.

After each round of the Delphi study, the agreement for each statement was quantitatively analyzed and comments were qualitatively evaluated by blinded investigators. Group results and a summary were reported after every round to the expert panel. In addition, anonymous individual feedback was provided separately to each expert [6].

Positive expert consensus was defined as a median score $\geq 7$ and no disagreement (30\% of the experts rated between 1 and 3 and simultaneously $30 \%$ between 7 and 9). Negative expert consensus was described as a median score $\leq 3$ and no disagreement.

If an item achieved positive expert consensus it was no longer included in the next Delphi round for agreement evaluation. Minor revisions based on the experts' comments were still possible. The revisions were then included for comments in the next round. Items which have not reached positive consensus were considerably revised according to the experts' suggestions or excluded.

The round 1 survey included three parts. Part one asked about demographic characteristics of the experts and part two about general aspects of retropulsion. The third part focused on the Scale for Retropulsion-Vo and included 40 statements which were rated by the experts on a 9-point Likert Scale. The round 2 survey included 22 statements about the Scale for Retropulsion-V1.1 and the round 3 survey involved only one statement about the general opinion about the Scale for Retropulsion-V1.2. 
Participants and recruitment

Inclusion criteria for experts in this study were 1) working in the field of neurorehabilitation and/or geriatrics for at least two years, 2) a minimum of two years of experience with topics related to postural control, and 3) regular contact with subjects who show altered postural control. Potential candidates were identified based on publications about retropulsion, backward disequilibrium, or lateropulsion. They were recruited via an email, inviting them to participate in the online Delphi study. For non-responders, an e-mail reminder was sent after three weeks, followed by another reminder after approximately five weeks. All experts gave written informed consent before the start of the first Delphi round. The experts remained anonymous throughout the Delphi process. Experts who did not answer a survey were excluded from the subsequent Delphi rounds.

\section{Results}

\section{Expert panel}

Thirteen experts were included in the panel and invited to round 1 of the Delphi study. The flow of the experts through the study is shown in Figure 1.

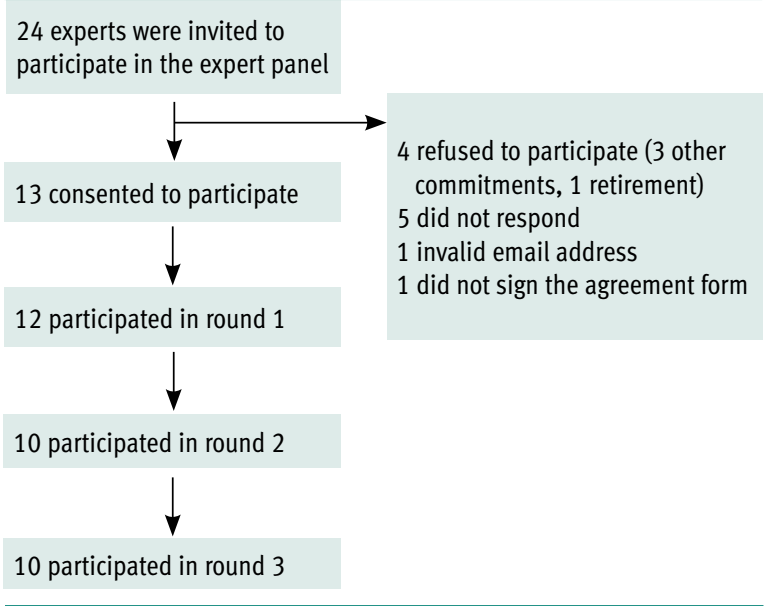

Fig. 1: Flow diagram showing the participation of experts in the Delphi study

The experts included in this study were from France $(n=4)$, Canada $(n=2)$, United States $(n=2)$, Australia $(n=2)$, Germany $(n=1)$, United Kingdom $(n=1)$ and involved different disciplines: medical doctor (3), physiotherapist (5), researcher (human movement science, biomechanics, psychologist) (3), kinesiologist (1). The overall working experience in neurorehabilitation, neurology, or/and geriatrics was on average $22.5 \pm 10.0$ years. Nine experts indicated experience in neurorehabilitation, 5 in neurology, 7 in geriatrics, 12 in postural control, 7 in falls, and 5 in spatial orientation.
General aspects about retropulsion

The experts rated the relevance of retropulsion high to very high for neurorehabilitation (median 7.5, IQR 2) and geriatrics (median 8, IQR 1.75). Disorders or impairments which were stated to be associated with retropulsion were neurological disorders in general, stroke, cerebellar problems, Parkinson's diseases, geriatric and older subjects, dementia and cognitive disorders, reduced alertness and attentional deficits, prolonged bed rest or long time periods in reclined positions, poor sensory acuity or perceptual deficits, risk of falling, poor balance, and ambulation problems.

While 5 experts indicated familiarity with scales or assessments for retropulsion, this was not the case for 7 experts. The former named the Backward Disequilibrium Scale, the Mini Motor Test, and the Pull test. There was disagreement between the experts about the limitation of existing scales (median 6, IQR 4). Overall, limitations of existing scales seem to be insufficient (evaluation of) clinimetric properties, lack of sensitivity, variability in the methods (pull test), lack of discriminatory power, dependency on the experience of the assessor, and absence of information for the elaboration of rehabilitation programs. There was agreement that a new scale to assess retropulsion is needed (median 7 , IQR 3). The experts mentioned the following issues that should be considered in a new scale: passive and active resistance, static postures (sitting and standing) and postural transitions (sit to stand, walking, and turning), weighting of retropulsion, influence of sensory deficits, and awareness.

\section{Delphi process}

The preliminary version of the Scale for Retropulsion which has been set up by an interdisciplinary team was based on literature, existing clinical scales (e.g. Burke Lateropulsion Scale [4], Pull test [12], Backward Disequilibrium Scale [10], and the Scale for Contraversive Pushing [8] and clinical experience. The Scale for Retropulsion-Vo comprised three categories (A. Static postural control, B. Reactive postural control, and C. Resistance) and included 11 items.

The three rounds of the Delphi study were conducted over the course of 5.5 months (March 2018 to August 2018). Data collection of each of the four rounds took about four weeks. The flow diagram for the Delphi process is shown in Figure 2.

\section{Round 1:}

Twelve of thirteen invited experts participated in Round 1 of the Delphi process. Out of the twelve experts, two had not completed the whole questionnaire.

Results of the round 1 survey can be found in supplement1. None of the statements about the Scale for 
Retropulsion-Vo showed disagreement. Positive expert consensus was achieved for 37 statements while three statements did not reach consensus (statement 16, 39 $\mathrm{a}-\mathrm{e}$, and $40 \mathrm{a}-\mathrm{e})$. Consequently, all items of subtest A "Static postural control" and subtest B "Reactive postural control” had reached positive consensus after round 1 , while this was not yet achieved for subtest $\mathrm{C}$ "Resistance".

As a result of the experts' comments, all items were slightly revised, even though they had already reached positive consensus. The revisions of these items mainly involved clarifications of the procedures and the instructions, and adjustments of the scoring (4-level scoring for all items). The items which had not yet reached consensus were considerably revised. Based on the experts' comments, also the structure of the scale was revised and a new subtest was introduced which comprises transferring and walking.

In addition, one issue of the general instructions had not reached consensus, namely the suggested procedure if an item cannot be done to a reason other than retropulsion. Based on discussion and literature review a modified solution was proposed and an example was given at the beginning of the scale to clarify the procedure.

Round 2:

Ten of twelve invited experts participated in the round 2 survey. Results of the round 2 survey are shown in supplement 2. No statement showed disagreement and all statements reached positive consensus after round 2 . Consequently all items of the four subtests showed consensus and no further evaluation of the items was required. Although there was an overall positive consensus, the scale was slightly revised as a result of the experts' comments. The revisions were included for comments in round 3 of the Delphi study. General satisfaction with the scale was high (median 8, IQR 1).

\section{Round 3:}

Ten of ten invited experts participated in the round 3 survey. Results of the round 2 survey can be found in supplement 3. The general agreement with the Scale for Retropulsion-V1.2 was very high (median 9, IQR 1).

The final version of the Scale for Retropulsion can be found in Appendix 1. It consists of four categories: A) static postural control, B) reactive postural control, C) resistance, and D) dynamic postural control. Each subtest is rated in a sitting (starting) position and in a standing (starting). The scale includes in total eight items which are arranged in the order of the body position to be tested (sitting and standing). Each item is scored on a 4-point rating scale (o no retropulsion to 4 severe retropulsion). The evaluation sheet of the Scale for Retropulsion was designed in a way that the subtests and body positions (sitting and standing) can be scored separately and as a total (Figure 3). The Scale for Retro-

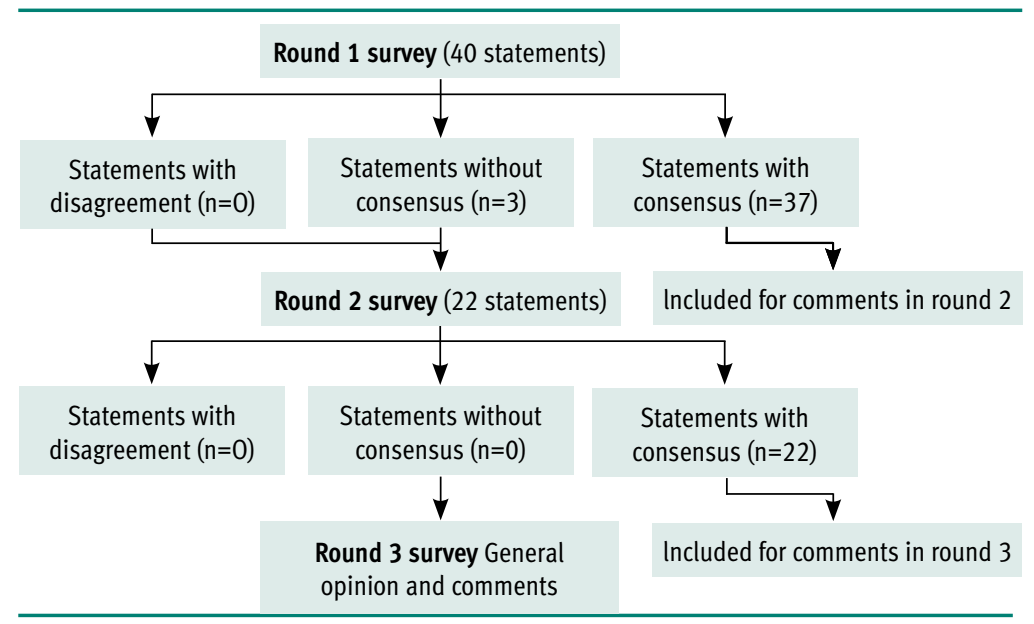

Fig. 2: Flow diagram showing the number and outcomes of statements in each Delphi round

\begin{tabular}{|c|c|c|c|c|}
\hline & & Sitting & Standing & Subscores \\
\hline A & Static postural control & $1 A=$ & $2 A=$ & $A: \_$(Max. 6) \\
\hline B & Reactive postural control & $1 B=$ & $2 B=$ & B : \\
\hline C & Resistance & $1 C=$ & $2 C=$ & $C: \quad($ Max. 6) \\
\hline \multirow[t]{2}{*}{ D } & Dynamic postural control & $1 D=$ & $2 \mathrm{D}=$ & $\mathrm{D}:{ }_{\square}(\operatorname{Max} .6)$ \\
\hline & & $\begin{array}{l}\text { Sitting: } \\
\text { (Max. 12) }\end{array}$ & $\begin{array}{l}\text { Standing: } \\
\text { (Max. 12) }\end{array}$ & $\begin{array}{l}\text { Total score: } \\
\end{array}$ \\
\hline
\end{tabular}

Fig. 3: Evaluation sheet of the Scale for Retropulsion

pulsion is designed as a bedside test, i.e. it can be tested in the patient's room and no special equipment was required. It takes about 5 to 15 minutes to complete the scale depending on the impairment level.

\section{Discussion}

We developed a clinical Scale for Retropulsion for individuals with neurological disorders that was modified and approved by experts within a Delphi study. The development process which was based on experts' opinion established the content validity of the scale. The level of international and multidisciplinary expert consensus was very high for the final version of the Scale for Retropulsion.

The four components which are evaluated by means of the Scale for Retropulsion in four subtests cover the different characteristics of retropulsion which we see in neurorehabilitation: spontaneous posterior body tilt with the tendency of backward falling, insufficient reactive postural responses, an active backward pushing with resistance against passive correction, and the difficulty to shift the center of mass actively forward [2, 3, 9, 10, 11, 13, 14]. The Scale for Retropulsion assesses sitting and standing, but also posture transition from sitting to standing and walking. We suppose that retropulsion compromises different postures and activities depending on the severity of the behavior, similar to lateropulsion [1]. 
The Delphi process represents a structured method for gathering opinions and achieving consensus when there is lack of evidence [5]. This Delphi study involved a heterogeneous expert panel from different disciplines and professions. Heterogeneous panels appear to make better quality decisions [7]. However, the rather small number of experts might have limited the stability of the results. When building up the panel, we focused on experts with high expertise in the field of retropulsion and related disorders to keep the quality high. As retropulsion is to date a rather neglected topic, only 24 experts where invited.

So far, research on retropulsion is very limited, amongst others due to the absence of an established tool to assess the behavior. It is suggested that the prevalence of retropulsion is quite high among elderly in an inpatient or outpatient clinical setting [14]. However, up to date there are no data available about the incidence and prevalence of retropulsion as well as about its time course.The Scale for Retropulsion provides clinicians, therapists and researchers with a clinical tool to evaluate and investigate postural instability in the sagittal plane. Clinical evaluation of the scale is now required in order to determine the clinimetric properties of the scale and its implementation in clinical practice and research.

\section{Summary}

Retropulsion represents a severe disorder of postural control in the sagittal plane. It is characterized by a posterior displacement of the center of mass with respect to the base of support and active resistance to passive correction of this posture.Although retropulsion seems quite frequent and relevant for neurorehabilitation, research on this postural behavior is limited. One major problem is that there is no established tool available to assess and quantify the behavior. Thus the objective of this Delphi study was to develop a clinical scale to assess retropulsion in neurological disorders. The Delphi method was applied to gather opinions from experts and build consensus on the validity of the content of theScale for Retropulsion. Twelve experts from different countries and disciplines participated in the Delphi study. The Delphi process comprised three rounds. All items of the scale reached consensus in the second round. The final version of the Scale for Retropulsion includes four subtests: A) static postural control, B) reactive postural control, C) resistance, and D) dynamic postural control which are tested in a sitting and a standing (initial) position. High level of international, multidisciplinary overall expert agreement was achieved for the scale (median 9, IQR 1). This bed side test will help clinicians, therapists and researchers to evaluate postural instability in the sagittal plane and to study the epidemiology, the etiology, and the rehabilitation process of patients with retropulsion. The next stage is to evaluate the clinimetric properties of the scale.

\section{Acknowledgements}

This work was supported by funds from the German Federal Ministry of Education and Research (BMBF IFB 01EOog01). We thank all the members of the expert panel (S. Babyar, I. Bonan, D. Brötz, C. Danells, J. Leif, A. Mansfield, J. Menant, F. Mourey, D. Pérennou, P. Sheets, D. Sturnieks, A. Yelnik) for their valuable contribution in this Delphi study.

\section{Reference}

1. Babyar SR, Peterson MG, Bohannon R, Perennou D, Reding M. Clinical examination tools for lateropulsion or pusher syndrome following stroke: a systematic review of the literature. Clinical rehabilitation 2009; 23(7): 639-50.

2. Barbieri G, Gissot AS, Perennou D. Ageing of the postural vertical. Age 2010; 32(1): 51-60.

3. Cardoen S, Santens P. Posterior pusher syndrome: A report of two cases. Clinical neurology and neurosurgery 2010; 112(4): 347-9.

4. D'Aquila MA, Smith T, Organ D, Lichtman S, Reding M. Validation of a lateropulsion scale for patients recovering from stroke. Clinical rehabilitation 2004; 18(1): 102-9.

5. Hohmann E, Brand JC, Rossi MJ, Lubowitz JH. Expert Opinion Is Necessary: Delphi Panel Methodology Facilitates a Scientific Approach to Consensus. Arthroscopy: the journal of arthroscopic \& related surgery: official publication of the Arthroscopy Association of North America and the International Arthroscopy Association 2018; 34(2): 349-51.

6. Jones J, Hunter D. Consensus methods for medical and health services research. Bmj 1995; 311(7001): 376-80.

7. Jorm AF. Using the Delphi expert consensus method in mental health research. Aust N Z J Psychiatry 2015; 49(10): 887-97.

8. Karnath HO, Ferber S, Dichgans J. The origin of contraversive pushing: evidence for a second graviceptive system in humans. Neurology 2000; 55(9): 1298-304.

9. Manckoundia P, Mourey F, Perennou D, Pfitzenmeyer P. Backward disequilibrium in elderly subjects. Clinical interventions in aging 2008; 3(4): 667-72.

10. Manckoundia P, Mourey F, Pfitzenmeyer P, Van Hoecke J, Perennou D. Is backward disequilibrium in the elderly caused by an abnormal perception of verticality? A pilot study. Clinical neurophysiology: official journal of the International Federation of Clinical Neurophysiology 2007; 118(4): 786-93.

11. Mourey F, Manckoundia P, Martin-Arveux I, Tavernier-Vidal B, Pfitzenmeyer P. Psychomotor Disadaptation Syndrome (PDS) A new clinical entity in geriatric patients. Geriatrics 2004; 59(5): 20-4.

12. Nonnekes J, Goselink R, Weerdesteyn V, Bloem BR. The retropulsion test: a good evaluation of postural instability in Parkinson's disease? J Parkinsons Dis 2015; 5(1): 43-7.

13. Pfitzenmeyer P, Mourey F, Tavernier B, Camus A. Psychomotor desadaptation syndrome. Archives of gerontology and geriatrics 1999; 28(3): 217-25.

14. Scheets PL, Sahrmann SA, Norton BJ, Stith JS, Crowner BE. What is backward disequilibrium and how do i treat it? A complex patient case study. Journal of neurologic physical therapy: JNPT 2015; 39(2): 119-26.

Conflict of interest:

The authors state that there is no conflict of interest.

\section{Correspondence to:}

Dr. Jeannine Bergmann

Schoen Clinic Bad Aibling and German Center for Vertigo and Balance Disorders

Ludwig-Maximilians University of Munich

Marchioninistrasse 15

81377 München

Jeannine.Bergmann@med.uni-muenchen.de 


\section{Appendix}

\section{Scale for Retropulsion}

Note: Although the masculine form is used for better legibility in this document, it always refers to both the female gender and the male gender alike.

\section{General instructions}

The Scale for Retropulsion can be tested in the patient's room and does not require any special testing materials. The scale includes four subtests: A) Static postural control, B) Reactive postural control, C) Resistance against passive movement, and D) Dynamic postural control. All subtests are first tested in a sitting (starting) position and thereafter in a standing (starting) position. The items are arranged in the order of the body positions to be tested (sitting and standing).

The scale evaluates postural behavior in the sagittal plane (spontaneous backward body tilt with the risk of backward falling, insufficient reactive postural responses, and active backward pushing with the inability to shift the center of mass forward, as well as resistance against passive correction), but not the patient's general ability to perform a task. This is why it is permitted to assist the patient as much as needed in the completion of the tasks. The assistance can be provided by the examiner and/or another person. The examiner only rates the assistance and the postural behavior in the sagittal plane, and not the assistance needed due to motor weakness, for example. The examiner's position is defined in the description of the individual item (e.g. behind the patient in subtest $\mathrm{B}$, on the paretic/more severely affected side of the body in subtests $C$ and D). However, if this position is not feasible or not safe enough, another position can be chosen. That position is then noted in the evaluation sheet.

Each item can be tested up to two times. No feedback about uprightness or performance should be given to the patient.

The scores are noted in the evaluation sheet which is designed in a way that the subtests and the body positions (sitting and standing) can be scored separately and as a total score.

The scale is not valid if a patient cannot sit despite being given maximal assistance and for any reasons other than retropulsion. In this case, the scale cannot be performed.

If the scale can generally be performed, but testing of individual items is not possible due to reasons other than retropulsion and despite maximum assistance, the examiner marks the corresponding item and notes down the reason in the evaluation sheet.
The item is then scored as follows in the evaluation sheet:

- For an item in the sitting (starting) position (1A-1D): The subtest cannot be scored.

- For an item in the standing (starting) position (2A2D): The item is scored o if the item is o in the sitting position in the corresponding subtest (no sign of retropulsion). The examiner gives it a score of 3 if the item is $\geq 1$ in the sitting position in the corresponding subtest.

For example: If static postural control in the sitting position was scored 2 (sitting is possible, but there is an increased tendency to fall backward), but the task cannot be tested in a standing position due to a very pronounced paresis, this item is given a score of 3 in the standing position. If, on the contrary, static postural control in the sitting position was scored o (stable, no falling backward), but the task cannot be tested in a standing position for a reason other than retropulsion, the item is given a score of $\mathrm{o}$ in the standing position. 


\section{Sitting}

Starting position for testing of the following subtests (1A-1D): Patient is sitting on the bed ${ }^{\star}$ (about $3 / 4$ of the femur should be supported by the sitting surface), the back is unsupported, the feet are resting on the floor, both hands are lying in the thighs, the eyes are open.

The examiner clarifies in advance that the patient has no orthopedic hip problems that could limit the hip flexion between $70^{\circ}$ and $120^{\circ}$ (e.g. joint prostheses, arthrosis of the hip, lower back pain).

*If a different support surface is used, please indicate here:

\begin{tabular}{|c|c|c|}
\hline & Task & Score \\
\hline $1 \mathrm{~A}$ & 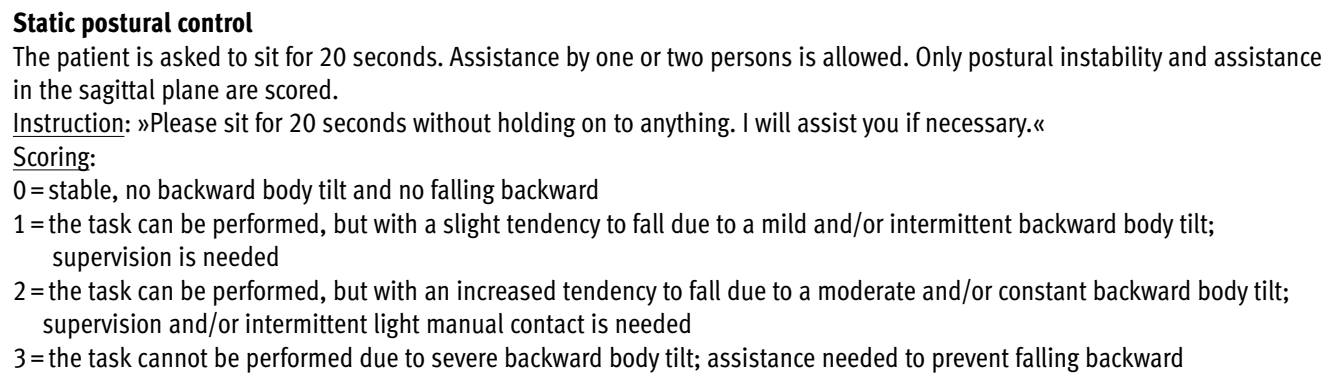 & $\begin{array}{l}1 A^{*} \\
\text { *If } 1 A=3 \\
\text { then score } \\
1 B=3\end{array}$ \\
\hline $1 \mathrm{~B}$ & 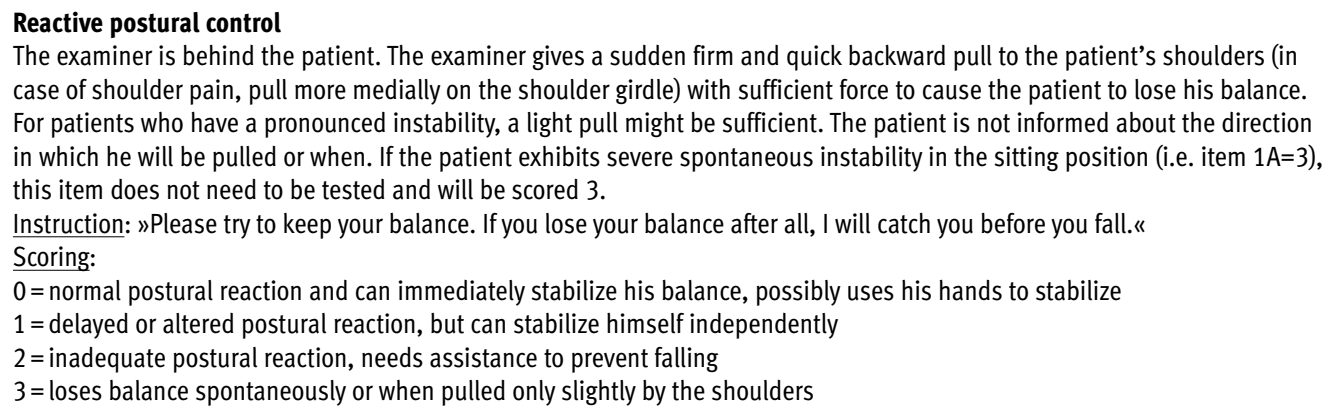 & $1 \mathrm{~B}$ \\
\hline $1 C$ & 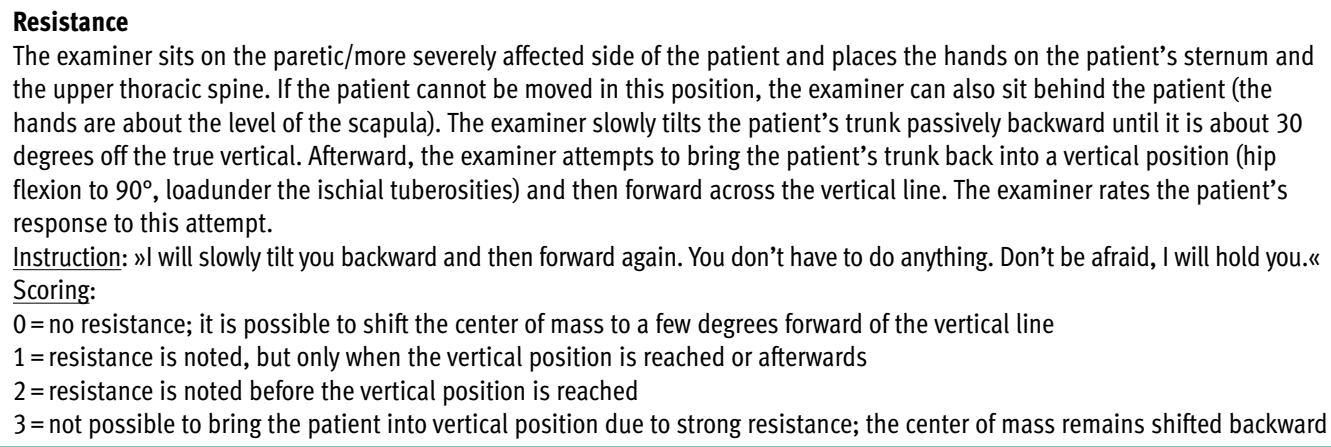 & $1 C$ \\
\hline 1D & 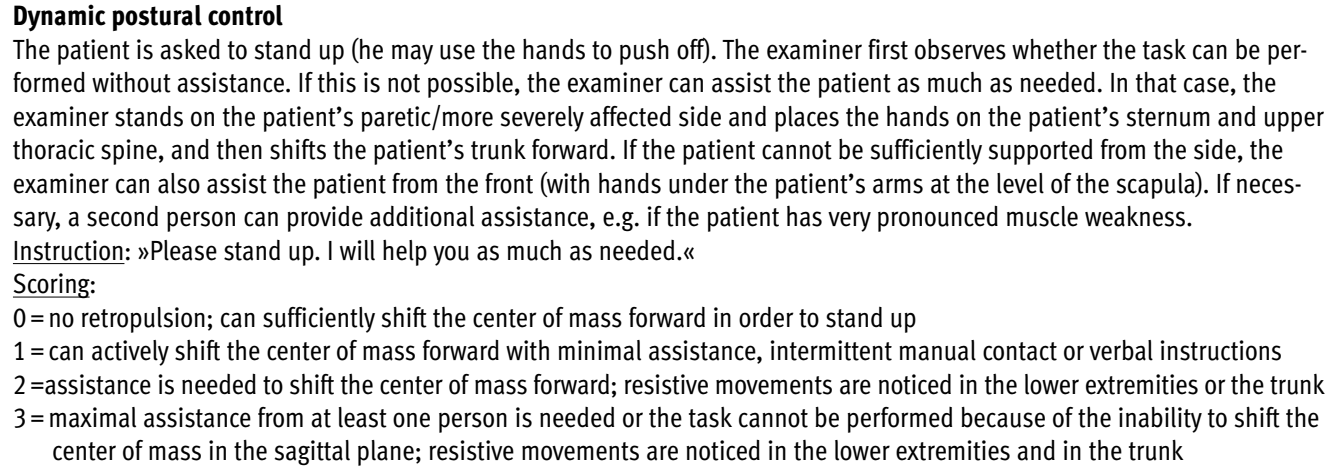 & $1 \mathrm{D}$ \\
\hline
\end{tabular}




\section{Standing}

Starting position for testing in the following subtests (2A-2D): Patient is standing with feet comfortably apart and parallel if possible.

\begin{tabular}{|c|c|}
\hline & Task \\
\hline $2 A$ & 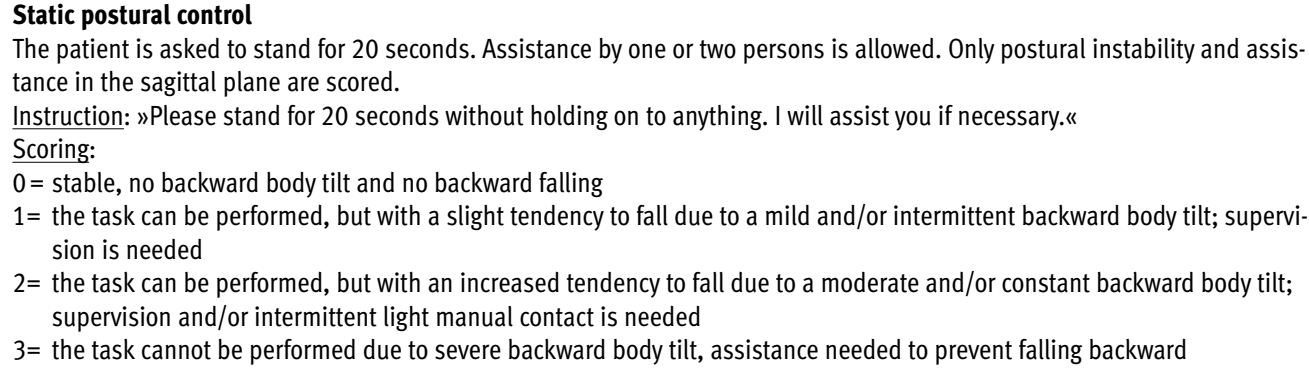 \\
\hline
\end{tabular}

2 B Reactive postural control

The examiner stands behind the patient. The examiner gives a sudden firm and quick backward pull to the patient's shoulders (in case of shoulder pain, pull more medially on the shoulder girdle) with sufficient force to cause the patient to lose his balance. For patients who have a pronounced instability, a light pull might be sufficient. The patient is not informed about the direction in which he will be pulled or when. If the patient exhibits severe spontaneous instability in the standing position (i. e. item $2 \mathrm{~A}=3$ ), this item does not need to be tested and will be scored 3 .

Instruction: »Please try to keep your balance. If you lose your balance after all, I will catch you before you fall.»

Scoring:

$0=$ normal postural reaction and can stabilize his balance independently, may take one or two steps or an ankle or hip reaction $1=$ three or more steps backward, but can stabilize himself independently

$2=$ inadequate postural reaction, needs assistance to prevent falling

$3=$ loses balance spontaneously or when pulled only slightly by the shoulders

\section{C Resistance}

Patient is standing with as much assistance as need. If possible, the entire soles of the feet are on the floor and the upper body is in an upright position. The examiner stands on the patient's paretic/more severely affected side and places the hands on the patient's sternum and the upper thoracic spine. If necessary, a second person can additionally provide assistance, e. g. in case of severe muscle weakness. If the patient cannot be moved in this position, the examiner can also stand behind the patient. The examiner slowly and passively shifts the patient's center of mass backward toward the heels. Afterward the examiner attempts to bring the patient back into a vertical position and forward toward the front of the feet. The examiner rates the patient's response to this attempt. The range of the motion is the length of the sole of the foot.

Instruction: »I will slowly tilt you backward and then forward again. You don't have to do anything. Don't be afraid, I will hold you." Scoring:

$0=$ no resistance, it is possible to shift the center of mass a few degrees forward of the vertical line and to bring load on to the entire foot (including the front of foot)

$1=$ resistance is noted, but only when the vertical position is reached or afterward (when the weight is shifted onto the forefoot) $2=$ resistance is noted before the vertical position is reached

$3=$ not possible to bring the patient into a vertical position due to strong resistance; the center of mass remains shifted backward

2D Dynamic postural control

The patient is asked to walk 4 meters. The examiner first observes whether the task can be performed without assistance. If this is not possible, the examiner can assist the patient as much as needed. In that case the examiner stands on the patient's paretic/more severely affected side and places the hands on the patient's sternum and upper thoracic spine, and then assists the patient in maintaining an upright body position and shifting the center of mass in the sagittal plane. If the patient cannot be sufficiently supported from the side, the examiner can choose different position and/or have a second person provide assistance.

Instruction: „Please try to walk up to 4 meters. I will help you as much as needed."

Scoring:

$0=$ no retropulsion, can independently maintain a forward progression of the center of mass and bring load on to the whole foot

$1=$ can actively maintain a forward progression of the center of mass with minimal assistance, intermittent manual contact or verbal instructions

$2=$ assistance is needed to bring the body in a vertical position and to shift the center of mass forward; slight resistance is noticed

$3=$ maximal assistance from at least one person is needed or the task cannot be performed because of the inability to bring the body in a vertical position and to shift the center of mass in the sagittal plane; resistive movements are noticed

\section{Score}

$2 \mathrm{~A}$

*If $2 A=3$

then score

$2 \mathrm{~B}=3$

$2 B$

$2 C$ 


\section{Evaluation sheet}

If an item cannot be tested for a reason other than retropulsion, mark it and note down the reason why it was not possible to do the testing.

The scoring of an item that cannot be tested for reasons other than the retropulsion and despite maximum allowed assistance is as follows:

- For an item in the sitting (starting) position (1A-1D): The subtest cannot be scored.

- For an item in the standing (starting) position (2A-2D): The item is scored o if the item is o in the sitting position in the corresponding subtest (no sign of retropulsion). The examiner gives it a score of 3 if the item is $\geq 1$ in the sitting position in the corresponding subtest.

\begin{tabular}{|c|c|c|c|c|}
\hline & & Sitting & Standing & Subscores \\
\hline A & Static postural control & $1 A=$ & $2 A=$ & $A: \_$(Max. 6) \\
\hline B & Reactive postural control & $1 \mathrm{~B}=$ & $2 B=$ & B : __ (Max. 6) \\
\hline C & Resistance & $1 C=$ & $2 C=$ & $C: \_$(Max. 6) \\
\hline \multirow[t]{2}{*}{ D } & Dynamic postural control & $1 \mathrm{D}=$ & $2 D=$ & $D: \_(\operatorname{Max} .6)$ \\
\hline & & $\begin{array}{l}\text { Sitting: } \\
\\
\end{array}$ & $\begin{array}{l}\text { Standing: } \\
\text { (Max. 12) }\end{array}$ & Total score: \\
\hline
\end{tabular}

Please note the reason why an item could not be tested:

Documentation of the examiner's position and the form and amount of assistance:

Main direction of retropulsion: $\square$ posterior $\square$ posterior-left $\square$ posterior-right 


\section{Supplement 1: Results round 1 Delphi study}

\begin{tabular}{|c|c|c|c|c|c|c|c|}
\hline \multicolumn{2}{|c|}{ Scale for Retropulsion - Vo } & Number of replies & Median & Percentile 25 & Percentile 75 & Disagreement & Consensus \\
\hline \multicolumn{8}{|c|}{ General aspects } \\
\hline 1. & $\begin{array}{l}\text { What is your general opinion of the Scale for Retropulsion-V0 (1=very unsatisfactory, } 9=\text { very } \\
\text { satisfactory)? }\end{array}$ & 10 & 7 & 6 & 7 & No & Yes \\
\hline 2. & The Scale for Retropulsion-V0 includes all clinically important characteristics of retropulsion. & 10 & 7 & 6 & 9 & No & Yes \\
\hline 3. & $\begin{array}{l}\text { The three subscores of the scale (A. static postural control, B. reactive postural control, and C. } \\
\text { resistance) are appropriate. }\end{array}$ & 10 & 9 & 7 & 9 & No & Yes \\
\hline 4. & The order of the items arranged in the order of the tested body position is appropriate. & 10 & 8 & 8 & 9 & No & Yes \\
\hline 5. & The structure of the scale is clear. & 10 & 9 & 8 & 9 & No & Yes \\
\hline 6. & The Scale for Retropulsion-V0 has an appropriate length and duration for testing. & 10 & 8 & 6 & 9 & No & Yes \\
\hline 7. & $\begin{array}{l}\text { The Scale for Retropulsion-V0 suits as a bed side test (difficulty, equipment, space require- } \\
\text { ments, etc.). }\end{array}$ & 10 & 8 & 7 & 9 & No & Yes \\
\hline 8. & The name "Scale for Retropulsion« is appropriate. & 10 & 9 & 7 & 9 & No & Yes \\
\hline 9. & General instructions of the scale are appropriate. & 10 & 7 & 4 & 8 & No & Yes \\
\hline 10. & $\begin{array}{l}\text { It is appropriate to include a different number of items in the subscores ( } 3 \text { items in A. static } \\
\text { postural control and B. reactive postural control, and } 5 \text { items in C. resistance). }\end{array}$ & 10 & 8 & 8 & 9 & No & Yes \\
\hline 11. & $\begin{array}{l}\text { It is appropriate that the scoring of the subscores results in a higher weighting of the resi- } \\
\text { stance subscore (score } 0-2 \text { for subscore } A \text {. static postural control and B. reactive postural con- } \\
\text { trol, and score } 0-3 \text { for subscore C. resistance). }\end{array}$ & 10 & 7 & 5 & 8 & No & Yes \\
\hline 12. & It is appropriate to score the subscores seperately and not in a total score. & 10 & 8 & 7 & 8 & No & Yes \\
\hline 13. & It is relevant to document a potential association with lateropulsion or pusher behavior. & 10 & 9 & 8 & 9 & No & Yes \\
\hline 14. & $\begin{array}{l}\text { It is appropriate to give no feedback about body orientation and patient's behavior during the } \\
\text { administration of the scale. }\end{array}$ & 10 & 9 & 9 & 9 & No & Yes \\
\hline 15. & It is appropriate to perform up to two trials to ensure appropriate scoring. & 10 & 8 & 8 & 9 & No & Yes \\
\hline 16. & $\begin{array}{l}\text { It is appropriate that, if an item cannot be done to a reason other than retropulsion and despi- } \\
\text { te maximum help allowed, the same score should be given as in the previous item of the same } \\
\text { subscore. }\end{array}$ & 10 & 5 & 4 & 7 & No & No \\
\hline \multicolumn{8}{|c|}{ Subscore A. Static postural control } \\
\hline 17. & $\begin{array}{l}\text { It is relevant to include backward falling due to backward body tilt in different static postures } \\
\text { in the Scale for Retropulsion. }\end{array}$ & 10 & 9 & 8 & 9 & No & Yes \\
\hline 18. & $\begin{array}{l}\text { It is appropriate to assess the subscale in the following three body positions: sitting with feet } \\
\text { on ground, sitting with feet off ground, and standing. }\end{array}$ & 10 & 8 & 7 & 9 & No & Yes \\
\hline 19. & The title of the subscale "static postural control" is appropriate. & 10 & 9 & 6 & 9 & No & Yes \\
\hline 20. & The scoring of the items (A1, A2, A3) from 0 to 2 with increasing severity is suitable. & 10 & 9 & 7 & 9 & No & Yes \\
\hline 21. & \multicolumn{7}{|l|}{ Item A1 - Static postural control during sitting with feet on the ground } \\
\hline 21.a) & Relevance & & 10 & 9 & 8 & 9 & No \\
\hline 21.b) & Procedure & & 10 & 8 & 7 & 9 & No \\
\hline 21.c) & Description of procedure & 10 & 9 & 7 & 9 & No & Yes \\
\hline 21.d) & Instruction & 10 & 9 & 7 & 9 & No & Yes \\
\hline 21.e) & Scoring & 10 & 9 & 7 & 9 & No & Yes \\
\hline 22. & \multicolumn{7}{|l|}{ Item A2 - Static postural control during sitting with feet off the ground } \\
\hline 22. a) & Relevance & 10 & 8 & 7 & 9 & No & Yes \\
\hline 22. b) & Procedure & 10 & 9 & 8 & 9 & No & Yes \\
\hline 22. c) & Description of procedure & 10 & 8 & 7 & 9 & No & Yes \\
\hline 22. d) & Instruction & 10 & 9 & 8 & 9 & No & Yes \\
\hline 22. e) & Scoring & 10 & 9 & 8 & 9 & No & Yes \\
\hline 23. & Item A3 - Static postural control during standing & & & & & & \\
\hline 23. a) & Relevance & 10 & 9 & 8 & 9 & No & Yes \\
\hline 23. b) & Procedure & 10 & 9 & 8 & 9 & No & Yes \\
\hline 23. c) & Description of procedure & 10 & 9 & 8 & 9 & No & Yes \\
\hline 23. d) & Instruction & 10 & 9 & 8 & 9 & No & Yes \\
\hline 23. c) & Scoring & 10 & 9 & 8 & 9 & No & Yes \\
\hline \multicolumn{8}{|c|}{ Subscore B - Reactive postural control } \\
\hline 24. & It is relevant to include reactive postural control in the Scale for Retropulsion. & 10 & 9 & 6 & 9 & No & Yes \\
\hline 25. & $\begin{array}{l}\text { It is appropriate to assess the subscale in the following three body positions: sitting with feet } \\
\text { on ground, sitting with feet off ground, and standing. }\end{array}$ & 10 & 7 & 5 & 9 & No & Yes \\
\hline 26. & The title of the subscale »reactive postural control« is appropriate. & 10 & 9 & 9 & 9 & No & Yes \\
\hline 27. & The scoring of the items (B1, B2, B3) from 0 to 2 with increasing severity is suitable. & 10 & 9 & 8 & 9 & No & Yes \\
\hline 28. & Item B1 - Reactive postural control during sitting with feet on the ground & & & & & & \\
\hline 28. a) & Relevance & 10 & 8 & 7 & 9 & No & Yes \\
\hline
\end{tabular}




\begin{tabular}{|c|c|c|c|c|c|c|c|}
\hline 28. b) & Procedure & 10 & 9 & 7 & 9 & No & Yes \\
\hline 28. c) & Description of procedure & 10 & 9 & 7 & 9 & No & Yes \\
\hline 28. d) & Instruction & 10 & 9 & 7 & 9 & No & Yes \\
\hline 28. e) & Scoring & 10 & 8 & 5 & 9 & No & Yes \\
\hline 29. & \multicolumn{7}{|l|}{ Item B2 - Reactive postural contol during sitting with feet off the ground } \\
\hline 29. a) & Relevance & 10 & 8 & 7 & 9 & No & Yes \\
\hline 29. b) & Procedure & 10 & 9 & 7 & 9 & No & Yes \\
\hline 29. c) & Description of procedure & 10 & 9 & 7 & 9 & No & Yes \\
\hline 29. d) & Instruction & 10 & 9 & 7 & 9 & No & Yes \\
\hline 29. e) & Scoring & 10 & 8 & 7 & 9 & No & Yes \\
\hline 30. & \multicolumn{7}{|l|}{ Item B3 - Reactive postural control during standing } \\
\hline 30. a) & Relevance & 10 & 9 & 8 & 9 & No & Yes \\
\hline 30. b) & Procedure & 10 & 9 & 8 & 9 & No & Yes \\
\hline 30.c) & Description of procedure & 10 & 9 & 7 & 9 & No & Yes \\
\hline 30. d) & Instruction & 10 & 9 & 7 & 9 & No & Yes \\
\hline 30.e) & Scoring & 10 & 8 & 5 & 9 & No & Yes \\
\hline \multicolumn{8}{|c|}{ Subscore C - Resistance } \\
\hline 31. & It is relevant to include resistance to passive correction in the Scale for Retropulsion. & 10 & 9 & 8 & 9 & No & Yes \\
\hline 32. & $\begin{array}{l}\text { It is appropriate to assess the scubscale in the following five body positions/posture tran- } \\
\text { sitions: sitting with feet on ground, sitting with feet off ground, standing, transferring, and } \\
\text { walking. }\end{array}$ & 10 & 8 & 5 & 9 & No & Yes \\
\hline 33. & The title of the subscale "resistance" is appropriate. & 10 & 9 & 7 & 9 & No & Yes \\
\hline 34. & The scoring of the items $(\mathrm{C} 1, \mathrm{C} 2, \mathrm{C} 3, \mathrm{C} 4, \mathrm{C} 5)$ from 0 to 3 with increasing severity is suitable. & 10 & 8 & 6 & 9 & No & Yes \\
\hline 35. & It is relevant to note the position of the examiner. & 10 & 8 & 7 & 9 & No & Yes \\
\hline 36. & \multicolumn{7}{|l|}{ Item C1- Resistance during sitting with feet on the ground } \\
\hline 36. a) & Relevance & 10 & 9 & 7 & 9 & No & Yes \\
\hline 36. b) & Procedure & 10 & 9 & 7 & 9 & No & Yes \\
\hline 36. c) & Description of procedure & 10 & 7 & 6 & 9 & No & Yes \\
\hline 36. d) & Instruction & 10 & 9 & 7 & 9 & No & Yes \\
\hline 36. e) & Scoring & 10 & 8 & 6 & 9 & No & Yes \\
\hline 37. & \multicolumn{7}{|l|}{ Item C2 - Resistance during sitting with feet off the ground } \\
\hline 37. a) & Relevance & 10 & 8 & 7 & 9 & No & Yes \\
\hline 37. b) & Procedure & 10 & 9 & 7 & 9 & No & Yes \\
\hline 37. c) & Description of procedure & 10 & 7 & 6 & 9 & No & Yes \\
\hline 37. d) & Instruction & 10 & 9 & 7 & 9 & No & Yes \\
\hline 37. e) & Scoring & 10 & 8 & 6 & 9 & No & Yes \\
\hline 38. & \multicolumn{7}{|l|}{ Item C3 - Resistance during standing } \\
\hline 38. a) & Relevance & 10 & 9 & 7 & 9 & No & Yes \\
\hline 38. b) & Procedure & 10 & 8 & 7 & 9 & No & Yes \\
\hline 38. c) & Description of procedure & 10 & 7 & 7 & 8 & No & Yes \\
\hline 38. d) & Instruction & 10 & 7 & 7 & 9 & No & Yes \\
\hline 38. e) & Scoring & 10 & 8 & 7 & 9 & No & Yes \\
\hline 39. & \multicolumn{7}{|l|}{ Item C4 - Resistance during transferring from sitting to standing } \\
\hline 39. a) & Relevance & 10 & 6 & 5 & 9 & No & Yes \\
\hline 39. b) & Procedure & 10 & 6 & 5 & 8 & No & No \\
\hline 39. c) & Description of procedure & 10 & 6 & 5 & 9 & No & No \\
\hline 39. d) & Instruction & 10 & 6 & 6 & 9 & No & No \\
\hline 39. e) & Scoring & 10 & 7 & 6 & 9 & No & No \\
\hline 40. & \multicolumn{7}{|l|}{ Item C5 - Resistance during walking } \\
\hline 40. a) & Relevance & 10 & 6 & 6 & 9 & No & No \\
\hline 40. b) & Procedure & 10 & 6 & 4 & 8 & No & No \\
\hline 40. c) & Description of procedure & 10 & 6 & 4 & 8 & No & No \\
\hline 40. d) & Instruction & 10 & 6 & 4 & 9 & No & No \\
\hline 40. d) & 40. e) Scoring & 10 & 6 & 4 & 9 & No & No \\
\hline
\end{tabular}




\section{Supplement 2: Results round 2 Delphi study}

\begin{tabular}{|c|c|c|c|c|c|c|}
\hline Scale for Retropulsion-V1 & Number of replies & Median & Percentile 25 & Percentile 75 & Disagreement & Consensus \\
\hline \multicolumn{7}{|l|}{ General aspects } \\
\hline 1. General opinion of the Scale for Retropulsion-V1. & 10 & 8 & 7 & 8 & No & Yes \\
\hline $\begin{array}{l}\text { 2. The four subscores of the Scale for Retropulsion-V1 (A) static postural control, B) reactive postural } \\
\text { control, C) resistance), and D) dynamic postural control) are appropriate. }\end{array}$ & 10 & 9 & 7 & 9 & No & Yes \\
\hline 3. The structure of the scale is clear. & 10 & 9 & 8 & 9 & No & Yes \\
\hline 4. The order of the items, arranged in the order of these two body positions, is appropriate. & 10 & 9 & 9 & 9 & No & Yes \\
\hline $\begin{array}{l}\text { 5. It is appropriate to assess the subscores only in one sitting position, namely in sitting with feet on } \\
\text { the ground. }\end{array}$ & 10 & 9 & 8 & 9 & No & Yes \\
\hline 6. The Scale for Retropulsion-V1 has an appropriate length and duration for testing. & 10 & 9 & 9 & 9 & No & Yes \\
\hline 7. The descriptions of the tasks are clear. & 10 & 8 & 7 & 9 & No & Yes \\
\hline 8. A video is useful to clarify the implementation of the items. & 10 & 9 & 9 & 9 & No & Yes \\
\hline $\begin{array}{l}\text { 9. It is appropriate to have a 4-level scoring for all items of the scale (i.e. the same weighting for all } \\
\text { subscores). }\end{array}$ & 10 & 9 & 8 & 9 & No & Yes \\
\hline $\begin{array}{l}\text { 10. It is appropriate that the subscores and body position (sitting and standing) can be scored sepa- } \\
\text { rately and in total. }\end{array}$ & 10 & 9 & 9 & 9 & No & Yes \\
\hline 11. The documentation of the most prominent direction of retropulsion is appropriate. & 10 & 8 & 7 & 9 & No & Yes \\
\hline 12. The evaluation sheet is clear. & 10 & 8 & 8 & 9 & No & Yes \\
\hline \multicolumn{7}{|l|}{$\begin{array}{l}\text { 13. The procedure, if an item cannot be done to a reason other than retropulsion and despite the } \\
\text { maximum help allowed }\end{array}$} \\
\hline 13. a) ...is appropriate & 10 & 8 & 7 & 9 & No & Yes \\
\hline 13. b) ...is clear & 9 & 7 & 6 & 8 & No & Yes \\
\hline \multicolumn{7}{|l|}{ Subscore A) Static postural control } \\
\hline 14. The 4-level scoring is appropriate for thissubscore. & 10 & 9 & 8 & 9 & No & Yes \\
\hline \multicolumn{7}{|l|}{ Subscore B) Reactive postural control } \\
\hline 16. The 4-level scoring is appropriate for thissubscore. & 10 & 9 & 7 & 9 & No & Yes \\
\hline \multicolumn{7}{|l|}{ Subscore C) Resistance } \\
\hline 18. It is appropriate to evaluate the subscore "resistance" during sitting and standing. & 10 & 9 & 8 & 9 & No & Yes \\
\hline \multicolumn{7}{|l|}{ Subscore D) Dynamic postural control } \\
\hline 20. It is relevant to evaluate the inability to shift the COM forward during active functional tasks. & 10 & 8 & 7 & 9 & No & Yes \\
\hline 21. It is appropriate to assess transferring from sitting to standing and walking in this subscore. & 10 & 8 & 8 & 9 & No & Yes \\
\hline 22. The title of the subscore "dynamic postural control" is suitable. & 10 & 8 & 5 & 9 & No & Yes \\
\hline 23. The scoring of the items (D1, D2) from 0 to 3 with increasing severity is appropriate. & 10 & 9 & 7 & 9 & No & Yes \\
\hline \multicolumn{7}{|l|}{ Item D1 } \\
\hline 24. a) The description of the procedure is appropriate. & 10 & 7 & 7 & 8 & No & Yes \\
\hline 24. b) The instruction to the patients is appropriate. & 9 & 8 & 7 & 9 & No & Yes \\
\hline 24. c) The scoring is appropriate. & 10 & 8 & 7 & 9 & No & Yes \\
\hline \multicolumn{7}{|l|}{ Item D2 } \\
\hline $\begin{array}{l}\text { 25. a) The item walking (ability to maintain a forward progression of the center of mass during walking) } \\
\text { is relevant. }\end{array}$ & 10 & 8 & 7 & 8 & No & Yes \\
\hline 25. b) The description of the procedure is appropriate. & 9 & 7 & 7 & 8 & No & Yes \\
\hline 25. c) The instruction to the patients is appropriate. & 9 & 8 & 7 & 9 & No & Yes \\
\hline 25. d) The scoring is appropriate. & 10 & 8 & 7 & 8 & No & Yes \\
\hline
\end{tabular}

\section{Supplement 3: Results round 3 Delphi study}

\begin{tabular}{|c|c|c|c|c|c|c|}
\hline Scale for Retropulsion-V1.1 & Number of replies & Median & Percentile 25 & Percentile 75 & Disagreement & Consensus \\
\hline Please indicate your general opinion with the Scale for Retropulsion-V1.1. & 10 & 9 & 8 & 9 & No & Yes \\
\hline
\end{tabular}

